\title{
High frame rate multi-resonance imaging refractometry with distributed feedback dye laser sensor
}

\author{
Christoph Vannahme, Martin Dufva and Anders Kristensen
}

High frame rate and highly sensitive imaging of refractive index changes on a surface is very promising for studying the dynamics of dissolution, mixing and biological processes without the need for labeling. Here, a highly sensitive distributed feedback (DFB) dye laser sensor for high frame rate imaging refractometry without moving parts is presented. DFB dye lasers are low-cost and highly sensitive refractive index sensors. The unique multi-wavelength DFB laser structure presented here comprises several areas with different grating periods. Imaging in two dimensions of space is enabled by analyzing laser light from all areas in parallel with an imaging spectrometer. With this multi-resonance imaging refractometry method, the spatial position in one direction is identified from the horizontal, i.e., spectral position of the multiple laser lines which is obtained from the spectrometer charged coupled device (CCD) array. The orthogonal spatial position is obtained from the vertical spatial position on the spectrometer CCD array as in established spatially resolved spectroscopy. Here, the imaging technique is demonstrated by monitoring the motion of small sucrose molecules upon dissolution of solid sucrose in water. The omission of moving parts improves the robustness of the imaging system and allows a very high frame rate of up to $12 \mathrm{~Hz}$. Light: Science \& Applications (2015) 4, e269; doi:10.1038/lsa.2015.42; published online 10 April 2015

Keywords: distributed feedback laser; label-free imaging; laser sensor; refractive index sensor

\section{INTRODUCTION}

Studying the dynamics of small molecule motion upon dissolution and mixing with label-free techniques as well as fast and multiplexed label-free biosensing are of considerable interest for the understanding of chemical, microfluidic and biological processes. While labeling, especially fluorescent labeling, is often used for visualizing small molecules these labels can affect the diffusion, convection and chemical properties of the molecules. Stimulated Raman scattering and stimulated emission microscopy techniques are label-free, but their imaging frame rate is limited due to the need for scanning. ${ }^{1}$ Among label-free biosensors, those based on optical transduction have proven most sensitive. ${ }^{2,3}$ Several optical sensors allow spatially resolving the refractive index on surfaces in microscope like set-ups. This imaging refractometry has e.g., been applied to detect nanoparticles, ${ }^{4,5}$ proteins, ${ }^{6-8}$ viruses $^{9-11}$ and cells ${ }^{12-16}$ and has enabled imaging as well as multiplexed sensing. ${ }^{10,17}$ In this context, label-free signal transduction is based on surface plasmon resonance, ${ }^{9,12,13,17,18}$ plasmonic hole arrays, ${ }^{19-23}$ photonic crystals ${ }^{5,14-16}$ and interferometry. ${ }^{8,10,11}$ Most of these methods rely on detecting a resonance shift in the optical spectrum. Imaging is often achieved by resolving optical resonances in one dimension of space with an imaging spectrometer. However, for imaging in two dimensions of space, the sample usually needs to be moved and images are assembled from multiple line scans. In this context, photonic crystal resonant reflectors have been used ${ }^{5,15}$ where scanning one line takes approximately $0.1 \mathrm{~s}$ which e.g., corresponds to a frame rate of $0.4 \mathrm{~Hz}$ for an image with 25 lines. In addition, moving the device relative to an imaging set-up is not only limiting the frame rate but can also affect the sample and is therefore undesired.

Dye-based distributed feedback (DFB) lasers are widely tunable laser light sources at visible wavelengths and have recently received attention as highly sensitive refractive index and biological sensors. ${ }^{24-28}$ They are inexpensive and feature very simple fabrication, low threshold and single-mode emission. Due to their narrow spectral linewidth and an optimized $^{29}$ high-sensitivity, nanoimprinted DFB dye laser sensors have proven to exhibit detection limits as low as $8 \times 10^{-6}$ RIU. $^{30}$

Here, a highly sensitive multi-wavelength nanoimprinted DFB dye laser sensor for high frame rate imaging refractometry without moving parts, enabling an imaging frame rate of up to $12 \mathrm{~Hz}$ is presented. The unique DFB laser structure comprises several areas of different grating periods which result in several spectrally separated laser emission peaks. This method is called multi-resonance imaging refractometry (Multi-RIR) in the following and is demonstrated by monitoring the complex motion of small sucrose molecules in water driven by dissolution, diffusion and convection.

\section{MATERIALS AND METHODS}

In this work, the Multi-RIR method is introduced with a surface emitting second-order DFB laser device with areas emitting at different emission wavelengths. The DFB laser wavelengths shift if the refractive index of the material on top of the device is changing. This way, the wavelength shift is used as a sensor signal giving the refractive index and refractive index changes on the surface. This relation is well understood 
and the refractive index of material on the surface can be calculated from the emission wavelength. ${ }^{30}$ For explanation of the Multi-RIR technique, a simplified device is shown in Figure 1a. This device comprises two areas emitting at two wavelengths $\lambda_{1}$ and $\lambda_{2}$. These wavelengths are spectrally close to each other but well separated as can be seen from the spectrum in Figure 1a. With an optical microscope system, a magnified image of the device surface is projected to the focal plane of the microscope where usually a charged coupled device (CCD) array is placed. Here, instead of a CCD array the entrance slit of an imaging spectrometer is placed in the focal plane. In addition, the image is compressed in $x$-direction with a cylindrical lens. Figure $1 \mathrm{~b}$ illustrates the resulting image obtained from the imaging spectrometer's CCD array. The vertical direction in this image is corresponding to the $y$-direction and is used for spatially resolving in this direction as commonly done with an imaging spectrometer. Here, for simplification only two rows of the CCD array are shown corresponding to two $y$-pixels. The horizontal direction in the spectrometer CCD array image corresponds to the wavelength. Due to the different wavelengths from the two areas at $x_{1}$ and $x_{2}$ two narrow lines appear in the image. These lines identify the positions $x_{1}$ and $x_{2}$, respectively. This is the key idea of the Multi-RIR method. The width $\Delta x$ of the areas with different resonant wavelength defines the spatial resolution of the imaging in $x$-direction. From the CCD image a refractometric image is obtained by finding the center wavelengths for each $y$-row and each laser line, i.e., $x$-position. Finding the center wavelength is based on fitting a Lorentzian function or on a center of mass approach. ${ }^{30}$ Refractive index changes can be detected as the wavelength of the lines is shifting which is indicated for the point in time $t=t_{1}$ in Figure 1b. While the method is explained here with only $2 \times 2$ pixels it is straight forward to extent it to many more pixels which is demonstrated in the following.

Figure 2a shows a schematic of a DFB dye laser device with 40 different regions with grating period $\Lambda_{i}$ (only three are shown in the schematic) ranging from $351 \mathrm{~nm}$ to $390 \mathrm{~nm}$ with an interval of $1 \mathrm{~nm}$ between the adjacent areas. The grating depth is approximately $100 \mathrm{~nm}$ and the duty cycle $25 \%$. The width of each area is $\Delta x=25 \mu \mathrm{m}$ and the whole nanostructured area is $1 \mathrm{~mm} \times 1 \mathrm{~mm}$. There is no gap between the areas.

The device was fabricated on a 0.5 -mm-thick 4 -inch Borofloat glass wafer where upon a thin film of Pyrromethene 597 (Exciton Technology Inc., Edmonton, Alberta, Canada ) doped Ormocomp mixed with ma-T 1050 thinner (25\% w/w; micro resist technology $\mathrm{GmbH}$, Berlin, Germany) was spin-coated to a thickness of approximately $400 \mathrm{~nm}$. A silicon stamp made by electron beam lithography and reactive ion etching and coated with an antistiction coating was used for replicating the grating structure via room temperature nanoimprint into the Ormocomp layer for $20 \mathrm{~min}$ at $10 \mathrm{kN}$. The Ormocomp film was cured by exposure to ultraviolet light and heated to $110{ }^{\circ} \mathrm{C}$ for $3 \mathrm{~min}$ before stamp and device were separated. Finally, $25 \mathrm{~nm}$ of $\mathrm{TiO}_{2}$ were ion beam deposited onto the Ormocomp film.

Figure $2 \mathrm{~b}$ schematically illustrates how the laser was positioned in a fluidic well consisting of 1-mm-thick piece of polymethyl methacrylate which was attached to the device surface with adhesive film. The well structure was filled with distilled water for investigation of the dissolution of solid sucrose which was added to the small well.

The optical set-up used for exciting the multiwavelength DFB laser device and analyzing its emission is illustrated in Figure $2 \mathrm{c}$. The DFB laser is excited with a nanosecond-pulse pump laser at $532 \mathrm{~nm}$ (CryLaS FDSS532-150; CryLaS GmbH, Berlin, Germany) which is triggered with a repetition rate of up to $12 \mathrm{~Hz}$. A dichroic mirror is reflecting the pump light onto the sample where it excites the DFB laser through a a
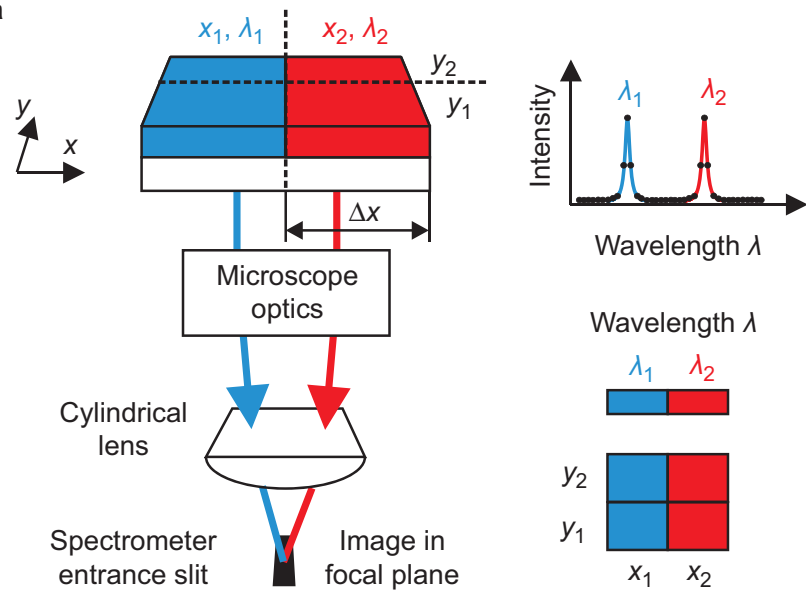

Wavelength $\lambda$

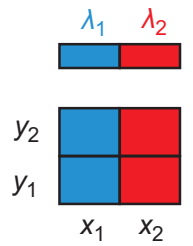

b Center wavelength finding

Spectrometer CCD image

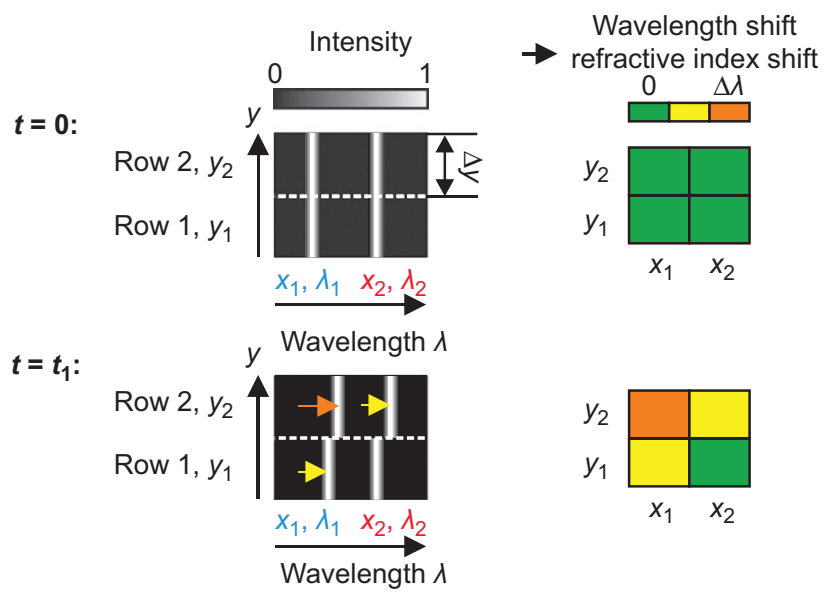

Figure 1 Operating principle of Multi-RIR. (a) The device, a DFB laser, consists of two laser areas that emit at two different center wavelengths, $\lambda_{1}$ (blue) and $\lambda_{2}$ (red). These two laser areas define the positions $x_{1}$ and $x_{2}$ of width $\Delta x$. A magnified image of the laser surface is projected at the same plane as the entrance slit of an imaging spectrometer. This image is compressed in $x$-direction by a cylindrical lens and this way light from both wavelength areas is coupled into the spectrometer. The $y$ direction is unaffected by the cylindrical lens. A schematic of the laser device from above shows the wavelengths of the emitted light from these areas indicated with blue and red color. (b) Schematic illustration of the images which are obtained from the spectrometer CCD array and corresponding refractometric images. For the point in time $t=0$, it is assumed that the same refractive index is present on top of the device at all positions. The emission from the two different areas results in two narrow lines in wavelength direction. As the $y$-direction is unaffected by the cylindrical lens, the $y$-position corresponds to the vertical direction in the imaging spectrometer CCD image. The spatial resolution in $y$-direction is affected by the magnification of the imaging and the number of pixels of the CCD array in this direction. Here, only two rows with pixels in $y$-direction are shown. Refractometric images, i.e., images of the surface refractive index changes, are obtained from the spectrometer CCD image by finding the center wavelength for each laser line and each row. It is assumed that at the point in time $t=t_{1}$ the refractive on top of the device has increased a certain amount at the pixel $(1,1)$ and less at the pixels $(1,2)$ and $(2,1)$ while it is unchained at pixel $(2,2)$. In this case, the center wavelengths shift due to the refractive index change as shown in the second spectrometer image. By finding the four center wavelengths from this CCD array image the refractometric image is obtained showing the wavelength shift and thus the refractive index shift for each of the four pixels. CCD, charged coupled device; DFB, distributed feedback; Multi-RIR, multi-resonance imaging refractometry. 
a

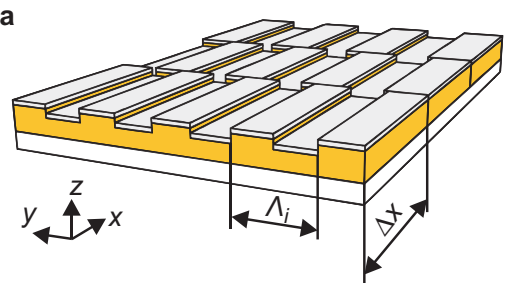

c

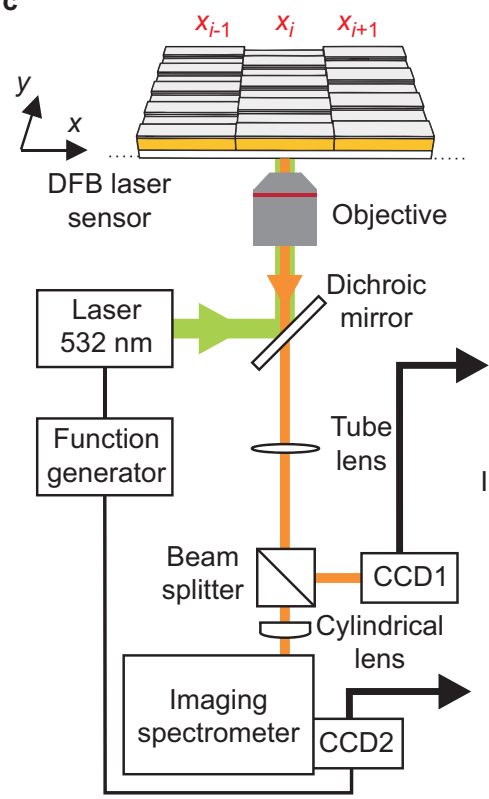

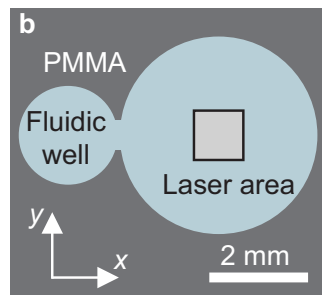

Spectrometer slit orientation
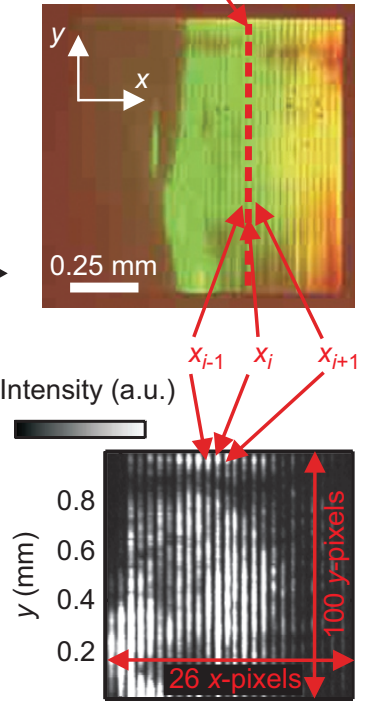

580

Wavelength $(\mathrm{nm})$

Figure 2 (a) Schematic illustration of DFB dye laser with areas of different grating period. Only three areas of different grating period are shown while the real device comprises forty areas. (b) Schematic of fluidic well structure. (c) Schematic of optical set-up with microscope color image of laser surface from CCD1 showing laser emission at different wavelengths and corresponding image from imaging spectrometer CCD array (CCD2) showing the different laser lines. CCD, charged coupled device; DFB, distributed feedback; PMMA, polymethyl methacrylate.

microscope objective with a pump fluence of typically $1-5 \mu \mathrm{Jm}^{-2}$. The pump beam was adjusted such that a spot with Gaussian shape with a beam waist of approximately $1.8 \mathrm{~mm}$ appeared on the surface in order to illuminate the $1 \mathrm{~mm} \times 1 \mathrm{~mm}$ large grating area homogeneously. The DFB laser emission of longer wavelength than the pump is transmitted through the dichroic mirror, while the mirror blocks the pump light. The emitted light is passing a tube lens and is split into two beams with a beam splitter. The first beam is used to create an image of the laser surface onto a CCD array, CCD1, for position control. The second beam is focused in $x$-direction with a cylindrical lens, while the $y$ direction is unaffected by this lens (compare Figure $2 \mathrm{c}$ for the directions relative to the device). Due to the compression with the cylindrical lens, light from all the laser areas is coupled into an imaging spectrometer. The CCD array of the imaging spectrometer (Acton SP-2756 imaging spectrograph with PIXIS100B digital CCD camera, $1340 \times 100$ pixels; Acton International, Ltd., Lincoln, NE, USA) is triggered with the same signal as the pump laser. The optical system presented here is capable of operating with a frame rate of up to $12 \mathrm{~Hz}$ limited by the readout time of the spectrometer CCD array. The upper inset in Figure $2 \mathrm{c}$ shows an image from the color CCD1 where lasing at a specific wavelength different from the other areas' lasing wavelengths is observed for each

region while pure water is present on the laser surface here. The different resonance wavelengths can be seen from the color change from green to orange in $x$-direction. The optical system gives a $4 \times$ magnification such that in $y$-direction the image of the $1 \mathrm{~mm}$ long laser area fills exactly the length of the $4 \mathrm{~mm}$ long entrance slit of the imaging spectrometer. Corresponding data from the imaging spectrometer CCD2 is presented in the lower inset of Figure 2c. The emission from the areas with different grating period is separated to several lines on the CDD image due to the varying wavelength, compare Figure $1 \mathrm{~b}$. If the refractive index of the laser's cladding is changing, the wavelength is shifting and this can be monitored by spectral shifts of the laser lines for all positions. By continuously taking images, the wavelength shift of each line is calculated and refractive index changes are monitored in time and two dimensions of space with the Multi-RIR method.

\section{RESULTS AND DISCUSSION}

In the following, it is shown how the multiwavelength DFB laser is used to monitor how a piece of solid sucrose is added to the smaller fluidic well (Figure 2b) and is dissolving in water. This results in motion of sucrose molecules as the sucrose is dissolved in water while the size of the solid sucrose is decreasing with time. Sucrose molecules are moving driven by diffusion and convection. The combination of these different physical processes results in a sucrose motion, which would be complex to be reflected with a simulation. Influence on the molecule movement by the pump laser light or induced heat might be possible, but is not expected as the total pump power is low and distributed homogeneously.

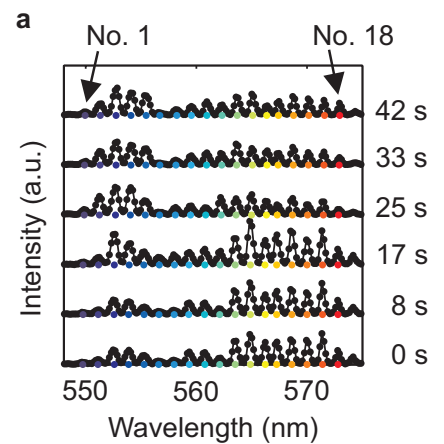

C

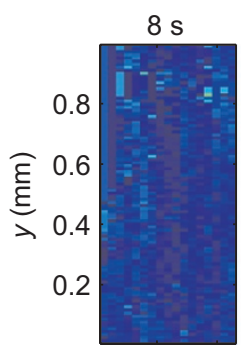

$0.2 \quad 0.4$

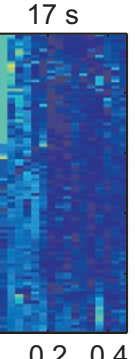

0.20 .4

\section{b}

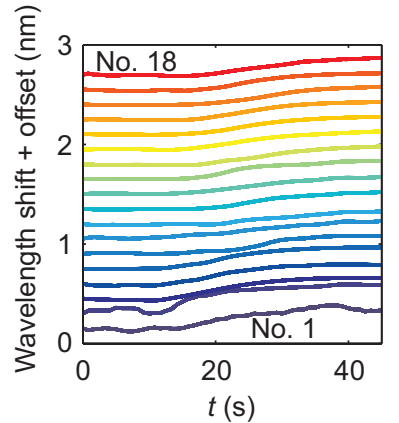

Wavelength shift (nm)
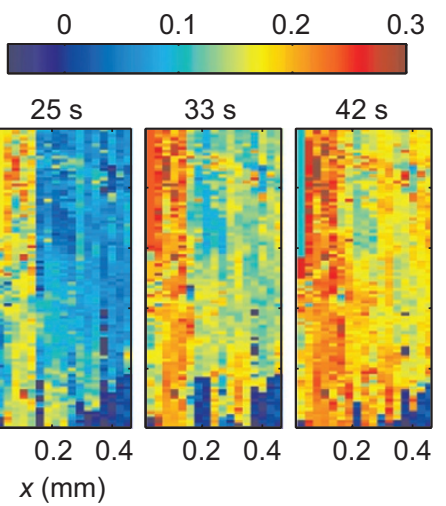

Figure 3 Label-free imaging with moderate spectral resolution. (a) Spectra for $y=0.65 \mathrm{~mm}$ for six different points in time (black symbols and lines). Colored symbols indicate the spectral position of the fitted center wavelengths. (b) Wavelength shift upon sucrose motion for $y=0.65 \mathrm{~mm}$. (c) Label-free images of sucrose motion (compare Supplementary Movies 1 and 2). 
Figure 3 illustrates how a laser was used to image the sucrose concentration change using 18 adjacent laser areas with a frame rate of $12 \mathrm{~Hz}$. Here, the spectrometer was used with a grating with $150 \mathrm{~g} \mathrm{~mm}^{-1}$ resulting in a spectral bandwidth of $0.17 \mathrm{~nm}$ corresponding to the length of one of the 1340 pixels in the wavelength direction of the $1340 \times 100$ pixel CCD array. Accordingly, there are 100 pixels in the $y$-direction. The single lasing peaks were found with a Matlab-based code from the data obtained with CCD2. Firstly, a set of lasing peaks corresponding to all $x$-positions was found by averaging the spectra of all $y$-positions at $t=0$. Laser lines were identified by finding all the local maxima within one spectrum whereat a minimum distance between the maxima as well as a minimum peak height were taken into account. For each laser peak the central wavelength was determined by fitting a Lorentz function to the data. For the data set shown in Figure 3, seven data points have been used for each fit. The number of data points needs to be limited such that the emission peak from the next lasing area does not influence the fit. At the same time, the fit result is usually better the more data points of the actual peak are used. The laser emission center wavelengths were determined for every single $y$-position and all the frames using the same approach. For illustration of the method, the spectra at different points in time are shown for $y=0.65 \mathrm{~mm}$ in Figure 3a. The calculated center emission wavelengths for the peaks are shown as colored symbols. Sucrose was added while the wavelength was monitored and thus, the spectra for $0 \mathrm{~s}$ and $8 \mathrm{~s}$ represent lasing with pure water on top of the device which results in a stable wavelength. Due to motion of the sucrose molecules and the resulting concentration increase the emission wavelength is red shifting after approximately $14 \mathrm{~s}$ as shown in Figure $3 \mathrm{~b}$ for $y=0.65 \mathrm{~mm}$. The same data analysis is done for all $y$-positions. The 18 laser lines correspond to 18 adjacent areas with a width of $25 \mu \mathrm{m}$ and thus identify the $x$-coordinate. Using this, the wavelength shift is imaged as shown in Figure 3c. Here, it can be seen that the wavelength, and thus, the refractive index of the water sucrose solution, is increasing from left to right as the sucrose is added on the left. The stable wavelength level before sucrose is added confirms that wavelength blue shifts induced by temperature changes ${ }^{31,32}$ are weak for the devices used and can be neglected here. Some of the pixels at later points in time remain unchanged. Here, the intensity peaks where too weak to be clearly identified by the code. Videos showing the data from CCD2 and the center wavelength positions found with the code (Supplementary Movie 1) and the calculated wavelength shift (Supplementary Movie 2) are attached as supplementary material.

In the experiment presented in Figure 3 the detection limit $d l$ of the DFB dye laser sensor is limited by the spectral bandwidth to approximately $2 \times 10^{-4}$ RIU. The detection limit is calculated from the sensitivity $s=90 \mathrm{~nm} \mathrm{RIU}^{-1}$ of the laser sensor and the standard deviation $\sigma$ of the Lorentzian fit to $d l=2 \sigma / s$ with $\sigma=6 \mathrm{pm}$ for 200 pulses with pure water on top of the device. Further details about this calculation are described elsewhere. ${ }^{30,33}$

So far, the spectrometer was used with a grating with $150 \mathrm{~g} \mathrm{~mm}^{-1}$ resulting in a spectral bandwidth of $0.17 \mathrm{~nm}$. By using a grating with $1800 \mathrm{~g} \mathrm{~mm}^{-1}$, the spectral bandwidth can be increased such that one spectrometer pixel in wavelength direction corresponds to $12 \mathrm{pm}$. However, this implies that only 11 laser peaks fit into the whole spectral range corresponding to the length of the spectrometer CCD. Thus, there is a tradeoff between the possible number of imaging pixels in $x$-direction and the possible detection limit with this method. Figure 4 shows the results of a similar sucrose dissolving experiment as before, this time with higher spectral resolution and only 11 laser peaks used for analysis. Figure 4a shows spectra for selected points in time again and Figure $4 \mathrm{~b}$ shows the wavelength shift which in this case has been
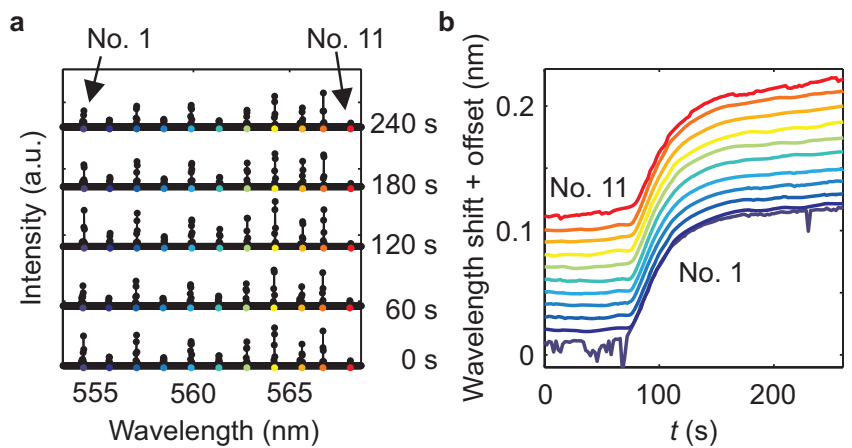

C

d

Wavelength shift $(\mathrm{nm})$

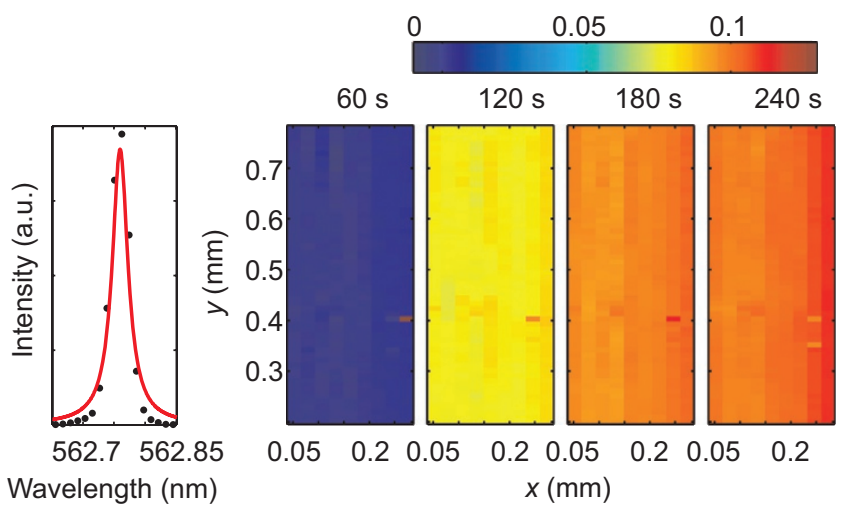

Figure 4 Label-free imaging with high spectral resolution. (a) Spectra for $y=0.65 \mathrm{~mm}$ for 6 different points in time (black symbols and lines). Colored symbols indicate the spectral position of the fitted center wavelengths. (b) Wavelength shift for $y=0.65 \mathrm{~mm}$. (c) CCD2 data and Lorentzian fit for peak No. 7. (d) Label-free images of sucrose motion (compare Supplementary Movie 3). CCD, charged coupled device.

corrected for a linear blue shifting of the wavelength caused by dye bleaching (compare Ref. 30 for bleaching effects). The blue shift has been calculated from the data for the first $80 \mathrm{~s}$ where pure water was present on top of the device. In general, there is less noise compared to the previous experiment due to the decreased spectral bandwidth and the detection limit is approximately $6 \times 10^{-5}$ RIU now. However, the noise increases for the peaks No. 1 and 11. A single peak and the Lorentzian fit are shown in Figure 4c. Again, seven data points have been used for fitting. In Figure 4d, images for different points in time reflecting the wavelength shift upon dissolving sucrose are shown. Due to the short total length in $x$-direction the wavelength shift difference between the $x$-positions may seem weak in comparison to the previous experiment. A corresponding movie (Supplementary Movie 3) is attached as supplementary material.

Wavelength shifts are shown here in order to illustrate the method, but the actual sucrose concentration can be calculated from the wellknown refractive index of sucrose-water solutions ${ }^{34}$ and the sensitivity

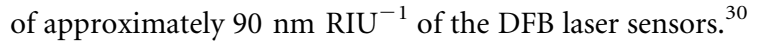

\section{CONCLUSIONS}

In summary, imaging refractometry with multiwavelength DFB dye lasers with a frame rate of $12 \mathrm{~Hz}$ has been demonstrated introducing the Multi-RIR method. The main advantages of this technique are the low detection limit of the DFB dye laser sensor, the high imaging frame rate mainly limited by the spectrometer CCD, and that the use of moving parts is avoided. In addition, the presented method of using varying 
spectral resonances for spatial resolution in one direction is promising for other optical resonant structures. The use of passive optical resonances would avoid the need for a pump laser source and the undesirable effects of bleaching blue shifts while it would also result in higher detection limits. With the presented method, the spatial resolution in $x$-direction is defined by the width of the areas of varying grating period $\Delta x$. It can be increased by using a smaller $\Delta x$, but as the number of areas that can be analyzed is limited, this will also limit the overall size of the whole analyzed area in $x$-direction. In $y$-direction, the spatial resolution is limited because of the coupled nature of the laser light. With passive photonic crystals, the spatial resolution can be down to $3.5 \mu \mathrm{m}$ in $y$-direction. ${ }^{35}$

The Multi-RIR technique presented here could pave the way towards label-free analysis of e.g., signaling molecule diffusion. Especially the low detection limit combined with high frame rate imaging opens up for real-time analysis for a variety of chemical, microfluidic, and biophysical applications. In addition, the optical set-up offers the possibility of multiplexed analysis of a large number of analysis spots with high frame rate.

\section{ACKNOWLEDGEMENTS}

The authors thank JS Clausen for assistance with electron beam lithography and CLC Smith, KT Sørensen and E Højlund-Nielsen for fruitful discussions. CV acknowledges support from the Danish Research Council for Technology and Production Sciences (Grant No. 12-126676).

1 Min W. Label-free optical imaging of nonfluorescent molecules by stimulated radiation. Curr Opin Chem Biol 2011; 15: 831-837.

2 Fan X, White IM, Shopova SI, Zhu H, Suter JD et al. Sensitive optical biosensors for unlabeled targets: a review. Anal Chim Acta 2008; 620: 8-26

3 Fan X, White IM. Optofluidic microsystems for chemical and biological analysis. Nat Photonics 2011; 5: 591-597.

4 Nie S, Emroy RE. Probing single molecules and single nanoparticles by surfaceenhanced Raman scattering. Science 1997; 275: 1102-1106.

5 Zhuo Y, Hu H, Chen W, Lu M, Tian L et al. Single nanoparticle detection using photonic crystal enhanced microscopy. Analyst 2014; 139: 1007-1015.

6 Yao J, Stewart ME, Maria J, Lee TW, Gray SK et al. Seeing molecules by eye: surface plasmon resonance imaging at visible wavelengths with high spatial resolution and submonolayer sensitivity. Angew Chem Int Ed 2008; 47: 5013-5017.

7 Johansson B, Höök F, Klenerman D, Jönsson P. Label-free measurements of the diffusivity of molecules in lipid membranes. Chemphyschem 2014; 15: 486-491.

8 Arroyo JO, Andrecka J, Spillane KM, Billington N, Takagi Y et al. Label-free, all-optical detection, imaging, and tracking of a single protein. Nano Lett 2014; 14: 2065 2070.

9 Wang S, Shan X, Patel U, Huang X, Lu J et al. Label-free imaging, detection, and mass measurement of single viruses by surface plasmon resonance. Proc Natl Acad Sci USA 2010; 107: 16028-16032.

10 Lopez CA, Daaboul GG, Vedula RS, Özkumur E, Bergstein DA et al. Label-free, multiplexed virus detection using spectral reflectance imaging. Biosens Bioelectron 2011; 26: 3432-3437.

11 Daaboul GG, Yurt A, Zhang X, Hwang GM, Goldberg BB et al. High-throughput detection and sizing of individual low-index nanoparticles and viruses for pathogen identification. Nano Lett 2010; 10: 4727-4731.

12 Jamil MM, Denyer MC, Youseffi M, Britland ST, Liu S et al. Imaging of the cell surface interface using objective coupled widefield surface plasmon microscopy. J Struct Biol 2008; 164: 75-80.
13 Peterson AW, Halter M, Tona A, Bhadriraju K, Plant AL. Surface plasmon resonance imaging of cells and surface-associated fibronectin. BMC Cell Biol 2009; 10: 16.

14 Lidstone EA, Chaudhery V, Kohl A, Chan V, Wolf-Jensen T et al. Label-free imaging of cell attachment with photonic crystal enhanced microscopy. Analyst 2011; 136: 3608-3615

15 Chen W, Long KD, Lu M, Chaudhery V, Yu H et al. Photonic crystal enhanced microscopy for imaging of live cell adhesion. Analyst 2013; 138: 5886-5894.

16 Nazirizadeh Y, Reverey J, Geyer U, Lemmer U, Selhuber-Unkel C et al. Material-based three-dimensional imaging with nanostructured surfaces. App/ Phys Lett 2013; 102: 011116.

17 Danz N, Kick A, Sonntag F, Schmieder S, Höfer B et al. Surface plasmon resonance platform technology for multi-parameter analyses on polymer chips. Eng Life Sci $2011 ; 11: 566-572$

18 Huang B, Yu F, Zare RN. Surface plasmon resonance imaging using a high numerical aperture microscope objective. Anal Chem 2007; 79: 2979-2983.

19 Yang Y, Turnbull G, Samuel ID. Hybrid optoelectronics: a polymer laser pumped by a nitride light-emitting diode. Appl Phys Lett 2008; 92: 163306.

20 Cuiffi J, Soong R, Manolakos S, Mohapatra S, Larson D. Nanohole array senso technology: multiplexed label-free protein binding assays. Proc IFMBE 2010; 32: 572-575.

21 Kim K, Yajima J, Oh Y, Lee W, Oowada S et al. Nanoscale localization sampling based on nanoantenna arrays for super-resolution imaging of fluorescent monomers on sliding microtubules. Small 2012; 8: 892-900.

22 Gao Y, Xin Z, Zeng B, Gan Q, Cheng X et al. Plasmonic interferometric sensor arrays for high-performance label-free biomolecular detection. Lab Chip 2013; 13: 47554764.

23 Ciminelli C, Campanella CM, Dell'Olio F, Campanella CE, Armenise MN. Label-free optical resonant sensors for biochemical applications. Prog Quantum Electron 2013; 37: 51-107.

24 Lu M, Choi SS, Wagner CJ, Eden JG, Cunningham BT. Label free biosensor incorporating a replica-molded, vertically emitting distributed feedback laser. App/ Phys Lett 2008; 92: 261502

25 Lu M, Choi SS, Irfan U, Cunningham BT. Plastic distributed feedback laser biosensor. Appl Phys Lett 2008; 93: 111113.

26 Ge C, Lu M, Zhang W, Cunningham BT. Distributed feedback laser biosensor incorporating a titanium dioxide nanorod surface. Appl Phys Lett 2010; 96: 163702.

27 Grivas C, Pollnau M. Organic solid-state integrated amplifiers and lasers. Laser Photonics Rev 2012; 6: 419-462.

28 Heydari E, Buller J, Wischerhoff E, Laschewsky A, Döring S et al. Label-Free biosensor based on an all-polymer DFB laser. Adv Opt Mater 2014; 2: 137-141.

29 Vannahme C, Smith CL, Christiansen MB, Kristensen A. Emission wavelength of multilayer distributed feedback dye lasers. App/ Phys Lett 2012; 101: 151123.

30 Vannahme $\mathrm{C}$, Leung $\mathrm{MC}$, Richter $\mathrm{F}$, Smith $\mathrm{CL}$, Hermannsson PG et al. Nanoimprinted distributed feedback lasers comprising $\mathrm{TiO}_{2}$ thin films: design guidelines for high performance sensing. Laser Photonics Rev 2013; 7: 1036-1042.

31 Zhao Z, Mhibik O, Leang T, Forget S, Chénais S. Thermal effects in thin-film organic solid-state lasers. Opt Express 2014; 22: 30092-30107.

32 Christiansen MB, Bu $\beta$ T, Smith CL, Petersen SR, Jørgensen MM et al. Single mode dye-doped polymer photonic crystal lasers. J Micromech Microeng 2010; 20: 115025.

33 White IM, Fan X. On the performance quantification of resonant refractive index sensors. Opt Express 2008; 16: 1020-1028.

34 Haynes WM, editor. CRC Handbook of Chemistry and Physics. 93rd ed. Boca Raton, FL: CRC Press; 2012.

35 Block ID, Mathias PC, Jones SI, Vodkin LO, Cunningham BT. Optimizing the spatial resolution of photonic crystal label-free imaging. Appl Opt 2009; 48: 6567-6574.

\section{(c) (i) (-) This work is licensed under a Creative Commons Attribution-}

BY NC SA NonCommercial-ShareAlike 3.0 Unported License. The images or other third party material in this article are included in the article's Creative Commons license, unless indicated otherwise in the credit line; if the material is not included under the Creative Commons license, users will need to obtain permission from the license holder to reproduce the material. To view a copy of this license, visit http://creativecommons.org/licenses/by-nc-sa/3.0/ 